\title{
Spatial integration in the broiler market in Peninsular Malaysia.
}

\begin{abstract}
The broiler sector in Peninsular Malaysia is marked by many structural elements of imperfect competition, particularly increasing vertical integration. This study adopts the Enders and Granger (1998) threshold autoregressive model to analyze market integration and pricing efficiency in the broiler sector. Results indicate that market integration exists in the broiler sector in spite of the structural rigidities that are present. However, asymmetries in price transmission are evident between the central market and regional wholesale markets.
\end{abstract}

Keyword: Cointegration; Livestock; Malaysia; Market integration; Threshold autoregression. 